\title{
High Serum Neuron-Specific Enolase Level Is Associated with Mild Cognitive Impairment in Patients with Diabetic Retinopathy
}

This article was published in the following Dove Press journal: Diabetes, Metabolic Syndrome and Obesity: Targets and Therapy

\section{Zi-Wei Yu* \\ Rong Liu (D) \\ Xin Li \\ Ying Wang \\ Yu-Hong Fu (D) \\ Hui-Yao Li \\ Yue Yuan \\ Xin-Yuan Gao}

Department of Endocrinology, The First Clinical Hospital of Harbin Medical University, Harbin 15000I, People's

Republic of China

*These authors contributed equally to this work
Correspondence: Xin-Yuan Gao

Tel +86 I390451700I

Email 15369337970@I63.com
Purpose: Diabetic retinopathy (DR) can increase the risk of mild cognitive impairment (MCI), which has been confirmed by previous researches. With the frequent occurrence of MCI in patients with DR, the early detection of MCI has become a research hot-spot. The aim of this study was to investigate the relationship between neuron-specific enolase (NSE) and MCI in patients with DR.

Patients and Methods: A total of 124 patients with DR, including $56 \mathrm{MCI}$ patients and 68 normal cognition patients, were recruited in this cross-sectional study. The demographic and clinical data of patients were collected through questionnaires. Serum NSE was measured using electrochemiluminescence immunoassay. The Minimum Mental State Examination (MMSE) scale was used to evaluate the cognitive function of the participants.

Results: Compared with the normal cognition group, serum NSE levels and HbA1c levels in the MCI group were higher, while MMSE scores and educational level were lower $(\mathrm{P}<0.05)$. Serum NSE levels were significantly negatively correlated with MMSE total score, attention and calculation score, and language score $(\mathrm{P}<0.05)$. After adjusting for confounding factors, serum NSE still increased the MCI risk in DR patients (OR:1.606, 95CI\%:1.264-2.041, $\mathrm{P}<0.001$ ). The areas under the receiver operating characteristics (ROC) curves (AUC) of the crude model and the adjusted model were 0.75 and 0.73 , respectively.

Conclusion: A high serum NSE level is an independent risk factor for MCI in DR patients. In addition, serum NSE is expected to be a potential biomarker in DR patients with MCI.

Keywords: neuron-specific enolase, mild cognitive impairment, type 2 diabetes mellitus, diabetic retinopathy

\section{Introduction}

According to statistics, there are about 50 million dementia patients around the world as of 2018. At present, one trillion dollars are used for the treatment of dementia patients, and the number will rise to two trillion dollars by $2030 .^{1}$ Dementia has caused tremendous pressure on the families and the society all over the world and has become an important disease that should not be overlooked. Mild cognitive impairment (MCI) is a transitional stage between normal aging and dementia and is also considered as a risk factor for dementia. ${ }^{2}$ Therefore, timely identification of MCI may be helpful to prevent dementia. Diabetic retinopathy (DR) is the most common microvascular complication of type 2 diabetes mellitus (T2DM) and the main cause of blindness in patients with T2DM. A recent cohort study based on 682 participants found that patients with any DR had twice the risk 
of developing cognitive impairment as those without DR. Surprisingly, this risk was three times higher in patients with moderate and severe DR than in those without DR. ${ }^{3}$ Based on these findings, more attention should be paid to the screening and intervention of mild cognitive impairment in T2DM patients with DR.

Neuron-specific enolase (NSE), a glycolytic enzyme, is located in the neurons and neuroendocrine cells, its physiological role is to regulate the growth and development of nerve cells. ${ }^{4}$ Interestingly, researchers found high levels of NSE in the serum of DR patients, and the levels of NSE were closely related to the severity of DR. ${ }^{5}$ Consistent with this, Asadova and his colleagues reported that compared with the control group, NSE was significantly increased in the serum and vitreous of patients with proliferative diabetic retinopathy (PDR). ${ }^{6}$ However, the cause of elevated serum NSE levels in DR patients remains unknown. Levels of NSE in serum and cerebrospinal fluid (CSF) are positively correlated with the damage of neurons, which can indirectly indicate the degree of nervous system injury. ${ }^{4}$ Therefore, it is considered as a sensitive marker of neuronal injury in many nervous system diseases. A vast number of studies have proved that excessive NSE levels are closely related to post-operative cognitive decline (POCD) in the elderly. ${ }^{7-9}$ Hence, this biomarker is expected to be a good indicator of mild cognitive impairment in patients with diabetic retinopathy.

Given that there is no study on the relationship between NSE and MCI in DR patients. The purpose of the present study was to clarify the association between serum NSE levels and MCI in Chinese DR patients.

\section{Patients and Methods}

\section{Subjects}

We recruited 124 T2DM patients with diabetic retinopathy who were treated in the endocrinology department of the First Affiliated Hospital of Harbin Medical University between March 2018 and February 2019. The diagnosis of T2DM in the subjects was consistent with the 1999 WHO diagnostic criteria. ${ }^{10}$ Each subject was examined by a clinical ophthalmologist with the use of an ophthalmoscope. The diagnosis and staging of DR were defined by fluorescein fundus angiography (FFA). Subjects who met any of the following conditions were excluded: (1) acute inflammatory diseases and autoimmune disease; (2) acute complications of diabetes mellitus; (3) neurological diseases that cause cognitive impairment (4) other ocular conditions that affect visual function; (5) patients with lung cancer, liver dysfunction, cardiac and respiratory failure; (6) patients with alcoholism and drug abuse; (7) patients with incomplete information. This study was approved by the Ethics Committee of the First Affiliated Hospital of Harbin Medical University and followed the Declaration of Helsinki and STARD guidelines. Written Informed consent was obtained from all participants.

\section{Clinical Data Collection}

A standard questionnaire was used to collect the information about age, diabetes duration, education level, lifestyle risk factors, medical history, diabetic complications, and diabetes therapy. BMI was calculated as weight $(\mathrm{kg})$ divided by the square of height $(\mathrm{m} 2)$. Laboratory tests of fasting blood sample were performed using standard biochemical analysis methods, which included glycosylated hemoglobin A1c (HbA1c), fasting C-peptide, total cholesterol (TC), triglycerides (TG), high density lipoprotein (HDL), low density lipoprotein (LDL), NSE, uric acid (UA), blood urea nitrogen (BUN) and creatinine $(\mathrm{Cr})$.

\section{Cognitive Function Assessment}

The diagnosis of MCI was based on the MCI diagnostic guidelines developed by the National Institute on Aging and the Alzheimer's Association workgroups: ${ }^{11}$ (1) a decline in cognition corroborated by an informant or a clinician; (2) objective evidence of Impairment in one or more cognitive domains, which was assessed by Mini-Mental State Examination (MMSE) in this present study; (3) preservation of independence in daily life; (4) not demented.

All subjects were tested with the MMSE scale. The MMSE scale can detect seven cognitive domains including orientation, registration, attention and calculation, recall, language, and visuospatial function. ${ }^{12}$ MCI is identified when an MMSE score is less than 27 and greater than 23.

\section{Statistical Analysis}

Statistical analysis was performed with SPSS version 25.0 and $\mathrm{R}$ software. The normally distributed variables were expressed as mean \pm standard deviation and were compared by $t$-test. The skewed variables were expressed as medians and were compared by using the Mann-Whitney $U$-test. Chi-square test was used to compare categorical variables. The relationship between the serum NSE levels and MMSE scores was examined by Spearman correlation analysis, partial correlation analysis, and linear regression analysis. Logistic regression analysis was used to explore the association between NSE 
and MCI in DR patients. Regression analysis with interaction term was used to investigate the effects of other variables on a relationship between NSE and MCI. The receiver operating characteristics (ROC) curve was constructed to evaluate the discrimination of NSE for MCI. The optimal cut-off point was determined by the maximum Youden index. A P-value $<0.05$ was regarded as statistically significant.

\section{Results}

\section{Baseline Characteristics}

The clinical and biochemical characteristics of the subjects are shown in Table 1 . There were 56 people in the MCI group
(23\%PDR, 34 men and 22 women) and 68 people in the normal cognition group (21\%PDR, 39 men and 29 women). Compared with the normal cognition group, the patients in the MCI group had higher levels of HbAlc and NSE but had a lower education level and MMSE total score $(\mathrm{P}<0.01)$. In addition, the scores of orientation, registration, attention and calculation, recall, and language in the MCI group were significantly lower than those in the normal cognition group $(\mathrm{P}<0.01)$. The two groups were similar in age, disease duration, BMI, fasting C-peptide, TC, TG, HDL, LDL, UA, $\mathrm{Cr}$, and $\mathrm{BUN}(\mathrm{P}>0.05)$. There were no significant differences between the two groups in diabetes treatment and the

Table I Clinical and Biochemical Characteristics Between DR Patients with Normal Cognition and DR Patients with MCI

\begin{tabular}{|c|c|c|c|}
\hline Variables & NCF Group $(N=68)$ & $\mathrm{MCl}$ Group (N=56) & P-value \\
\hline Age (years) & $55.50(50.25-63.00)$ & $57.50(48.50-62.75)$ & 0.964 \\
\hline Sex (Male,\%) & $39(57.3 \%)$ & $34(60.7 \%)$ & 0.705 \\
\hline Duration of DM (years) & $10.00(7.00-15.75)$ & $13.00(5.00-17.00)$ & 0.466 \\
\hline Current smoking (\%) & $26(38.2 \%)$ & 19 (33.9\%) & 0.620 \\
\hline Habitual alcohol drinking (\%) & $28(41.1 \%)$ & $24(42.8 \%)$ & 0.850 \\
\hline BMI (kg/m2) & $25.62 \pm 2.47$ & $25.64 \pm 2.86$ & 0.976 \\
\hline TG (mmol/L) & $1.83(1.23-2.65)$ & $2.13(1.49-3.24)$ & 0.073 \\
\hline NSE (ng/mL) & $11.80(10.15-13.88)$ & $14.55(12.00-17.57)$ & $<0.001$ \\
\hline Fasting C-peptide(ng/mL) & $1.20(0.90-1.70)$ & $1.40(0.73-2.00)$ & 0.796 \\
\hline $\mathrm{HbAIC}(\%)$ & $8.39 \pm 1.45$ & $9.42 \pm 2.16$ & 0.002 \\
\hline $\mathrm{TC}(\mathrm{mmol} / \mathrm{L})$ & $4.87(4.28-5.67)$ & $5.33(4.57-6.39)$ & 0.083 \\
\hline $\mathrm{HDL}(\mathrm{mmol} / \mathrm{L})$ & $1.21(1.10-1.38)$ & $1.28(1.13-1.52)$ & 0.173 \\
\hline $\mathrm{LDL}(\mathrm{mmol} / \mathrm{L})$ & $2.82(2.34-3.20)$ & $2.97(2.65-3.50)$ & 0.113 \\
\hline UA (umol/L) & 310.60 (272.30-379.68) & $322.25(262.15-407.38)$ & 0.984 \\
\hline $\mathrm{Cr}$ (umol/L) & $60.85(48.90-73.83)$ & $62.35(51.25-69.85)$ & 0.898 \\
\hline BUN (mmol/L) & $5.84 \pm 1.61$ & $6.15 \pm 1.99$ & 0.340 \\
\hline Education level (years) & $12.00(9-15)$ & $9.00(7.13-12.00)$ & 0.001 \\
\hline MMSE total score & $28.00(27.00-29.00)$ & $25.00(25.00-26.00)$ & $<0.001$ \\
\hline MMSE orientation score & $10.00(9.25-10.00)$ & $10.00(9.00-10.00)$ & 0.003 \\
\hline MMSE registration score & $3.00(3.00-3.00)$ & $2.00(2.00-3.00)$ & $<0.001$ \\
\hline MMSE attention and calculation score & $5.00(4.00-5.00)$ & $4.00(3.00-4.00)$ & $<0.001$ \\
\hline MMSE recall score & $3.00(2.25-3.00)$ & $2.00(2.00-3.00)$ & $<0.001$ \\
\hline MMSE language score & $8.00(8.00-9.00)$ & $7.00(7.00-8.00)$ & $<0.001$ \\
\hline History of hypertension(\%) & 30 (44.1\%) & $26(46.4 \%)$ & 0.797 \\
\hline Lower limb atherosclerosis (\%) & 54 (79.4\%) & 49 (87.5\%) & 0.232 \\
\hline Carotid atherosclerosis (\%) & $48(70.6 \%)$ & $4 \mathrm{l}(73.2 \%)$ & 0.746 \\
\hline Diabetic peripheral neuropathy(\%) & II (I6.2\%) & $16(28.5 \%)$ & 0.096 \\
\hline $\operatorname{PDR}(\%)$ & 21 (30.8\%) & $23(41.0 \%)$ & 0.238 \\
\hline Diabetic nephropathy (\%) & $16(23.5 \%)$ & 18 (32.1\%) & 0.285 \\
\hline Insulin use (\%) & $60(88.2 \%)$ & 50 (89.2\%) & 0.854 \\
\hline Metformin use (\%) & $43(63.2 \%)$ & $34(60.7 \%)$ & 0.773 \\
\hline Acarbose use (\%) & 9 (13.2\%) & $6(10.7 \%)$ & 0.668 \\
\hline DPP-4 inhibitor use (\%) & $24(35.2 \%)$ & 21 (37.5\%) & 0.799 \\
\hline Statins use (\%) & $33(48.5 \%)$ & 33 (58.9\%) & 0.248 \\
\hline
\end{tabular}

Abbreviations: DR, diabetic retinopathy; NCF, normal cognitive function; $\mathrm{MCl}$, mild cognitive impairment; DM, diabetesmellitus; BMI, body mass index; TG, triglyceride; NSE, neuron-specific enolase; HbAIc, hemoglobin AIc; TC, total cholesterol; HDL, high density lipoprotein; LDL, low density lipoprotein; UA, uric acid; $\mathrm{Cr}$, creatinine; BUN, blood urea nitrogen; MMSE, Minimum Mental State Examination; PDR, proliferative diabetic retinopathy. 
incidence of hypertension, lower limb atherosclerosis, carotid atherosclerosis, diabetic peripheral neuropathy, diabetic nephropathy and proliferative diabetic retinopathy $(\mathrm{P}>0.05)$.

\section{Associations Between Serum NSE Levels and MMSE Scores}

The Spearman correlation analysis demonstrated that serum NSE was significantly negatively correlated with MMSE scores, including total score $(\mathrm{r}=-0.421, \mathrm{P}<0.001)$, registration score $(\mathrm{r}=-0.237, \mathrm{P}=0.008)$, attention and calculation score $(\mathrm{r}=-0.294, \quad \mathrm{P}=0.001), \quad$ and language score $\quad(\mathrm{r}=-0.198$, $\mathrm{P}=0.028$ ). After adjusting age, sex, education level, and HbAlc, the associations between MMSE total score $(\mathrm{r}=-0.380, \quad \mathrm{P}<0.001)$, attention and calculation score $(\mathrm{r}=-0.272, \mathrm{P}=0.003)$, and serum NSE were attenuated, while the association between language score and serum NSE was enhanced $(\mathrm{r}=-0.222, \mathrm{P}=0.015)$. However, serum NSE levels did not correlate with the registration score $(p>0.05)$. Linear regression analysis showed that after adjustments for age, sex, education level, and $\mathrm{HbA1c}$, serum NSE was remained negatively correlated with MMSE total score $(\beta=-0.15, \mathrm{P}<0.001)$, attention and calculation score $(\beta=-0.06, P=0.003)$, and language score $(\beta=-0.042, \mathrm{P}=0.015)$. (Table 2$)$.

\section{Associations Between Serum NSE Levels and $\mathrm{MCl}$}

The associations between the serum NSE and MCI were summarized in Table 3. Univariate logical analysis indicated that high levels of NSE were correlated with an increased risk of cognitive impairment in patients with DR. Multivariate logistic regression analysis confirmed that the odds ratios (ORs) for MCI were 1.427 (95\% CI: 1.219-1.669, $\mathrm{P}<0.001)$ in the Model 2 adjusted for age and sex, 1.455 (95\% CI: 1.210-1.749, $\mathrm{P}<0.001$ ) in the Model 3 adjusted for age, sex, diabetes duration, education level, HbA1c, BMI,
TC, fasting C-peptide and UA. Further adjustment for lifestyle risk factors, history of hypertension, diabetic complications, and diabetes therapy (Model 4), the OR for MCI was 1.606 (95CI\%: 1.264-2.041, $\mathrm{P}<0.001$ ). These data suggested that the association between serum NSE and MCI was independent.

As shown in Table 4, diabetes duration, the increase in HbA1c, the degree of DR, and the occurrence of DPN were not modifiers of the association between NSE and MCI. ( $\mathrm{P}>0.05$ for interaction).

\section{Parameters for Diagnosing $\mathrm{MCl}$}

The area under the ROC curve (AUC) of the crude model was $0.75(95 \% \mathrm{CI}: 0.667-0.836, \mathrm{P}<0.001)$ and the AUC of the adjusted model was 0.73 (95\% CI:0.645 -0.819, $\mathrm{P}<0.001)$. The optimal cut-off point for serum NSE to diagnose MCI was 11.4 (sensitivity: 92.9\%, specificity: 47.1\%). The positive and negative predictive values were $59.1 \%$ and $88.9 \%$, respectively. The positive and negative likelihood ratios were 1.756 and 0.151 , respectively. (Figure 1 ).

\section{Discussion}

Up to now, the studies on the correlation between serum NSE and MCI are very limited. To our knowledge, there have been no studies on the association between serum NSE levels and MCI in DR patients. The main finding in this study was that high levels of serum NSE led to an increased risk of MCI in DR patients, particularly with poor performance in attention, calculation, and language. It is noteworthy that this association was not affected by other potential factors.

Normally, the NSE levels are very low in the serum. But when the nerve cells are damaged by ischemia, hypoxia or hypoperfusion, NSE can be released from the cells in large quantities and then enters the blood circulation through an injured blood-brain barrier, resulting in

Table 2 Associations Between Serum NSE Levels and MMSE Scores

\begin{tabular}{|c|c|c|c|c|c|c|}
\hline \multirow[t]{2}{*}{ Variables } & \multicolumn{2}{|c|}{$\begin{array}{l}\text { Spearman Correlation } \\
\text { Analysis }\end{array}$} & \multicolumn{2}{|c|}{$\begin{array}{l}\text { Partial Correlation } \\
\text { Analysis }\end{array}$} & \multicolumn{2}{|c|}{ Linear Regression Analysis } \\
\hline & $\mathbf{r}$ & P-value & $\mathbf{r}$ & P-value ${ }^{a}$ & $\mathrm{~B}(95 \% \mathrm{Cl})$ & P-value \\
\hline MMSE total score & -0.421 & $<0.001$ & -0.380 & $<0.001$ & $-0.150(-0.217--0.083)$ & $<0.001$ \\
\hline MMSE orientation score & -0.126 & 0.162 & -0.154 & 0.093 & $-0.026(-0.057-0.004)$ & 0.093 \\
\hline MMSE registration score & -0.237 & 0.008 & -0.101 & 0.274 & $-0.013(-0.035-0.01)$ & 0.274 \\
\hline MMSE recall score & -0.117 & 0.197 & -0.050 & 0.587 & $-0.007(-0.031-0.017)$ & 0.587 \\
\hline MMSE attention and calculation score & -0.294 & 0.001 & -0.272 & 0.003 & $-0.060(-0.099--0.021)$ & 0.003 \\
\hline MMSE language score & -0.198 & 0.028 & -0.222 & 0.015 & $-0.042(-0.076--0.008)$ & 0.015 \\
\hline
\end{tabular}

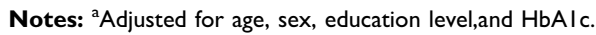

Abbreviations: NSE, neuron-specific enolase; MMSE, Minimum Mental State Examination. 
Table 3 Associations Between Serum NSE Levels and MCI

\begin{tabular}{|l|l|l|l|}
\hline & OR & $\mathbf{9 5 \%} \mathbf{~ C l}$ & P-value \\
\hline Model I & 1.428 & $1.220-1.671$ & $<0.001$ \\
Model 2 & 1.427 & $1.219-1.669$ & $<0.001$ \\
Model 3 & 1.455 & $1.210-1.749$ & $<0.001$ \\
Model 4 & 1.606 & $1.264-2.041$ & $<0.001$ \\
\hline
\end{tabular}

Notes: Model I: Crude model; Model 2: adjusted for age and sex; Model 3: Mode 2 +duration of DM, HbAlc,education level,BMI, UA, TC, and fasting C-peptide; Model 4: Model 3+current smoking, habitual alcohol drinking, insulin use, metformin use, statins use, carotid atherosclerosis, diabetic nephropathy, and diabetic peripheral neuropathy.

Abbreviations: NSE, neuron-specific enolase; $\mathrm{MCl}$, mild cognitive impairment; DM, diabetes mellitus; HbAlc, hemoglobin Alc; BMI, body mass index; UA, uric acid; TC, total cholesterol.

Table 4 Effects of Other Variables on the Relationship Between NSE and $\mathrm{MCl}$

\begin{tabular}{|l|l|l|l|l|}
\hline Characteristic & N & OR & $95 \%$ Cl & $\begin{array}{l}\text { P-value for } \\
\text { Interaction }\end{array}$ \\
\hline $\begin{array}{l}\text { Diabetes Duration (Years) } \\
<10 \\
\geq 10\end{array}$ & 50 & 1.426 & $1.127-1.804$ & 0.934 \\
\hline $\begin{array}{l}\text { HbAlc (\%) } \\
<7\end{array}$ & 74 & 1.445 & $1.166-1.791$ & \\
$7-9$ & 22 & 1.307 & $0.896-1.908$ & 0.745 \\
$\geq 9$ & 47 & 1.424 & $1.110-1.827$ & \\
\hline $\begin{array}{l}\text { Degree of DR } \\
\text { NPDR }\end{array}$ & 55 & 1.567 & $1.172-2.094$ & \\
PDR & 80 & 1.415 & $1.171-1.710$ & 0.831 \\
\hline $\begin{array}{l}\text { DPN } \\
\text { No }\end{array}$ & 44 & 1.469 & $1.104-1.953$ & \\
Yes & 97 & 1.441 & $1.199-1.731$ & 0.993 \\
\hline
\end{tabular}

Abbreviations: NSE, neuron-specific enolase; $\mathrm{MCl}$, mild cognitive impairment $\mathrm{HbAlc}$, hemoglobin AIc; DR, diabetic retinopathy; NPDR, non-proliferative diabetic retinopathy; PDR, proliferative diabetic retinopathy; DPN, diabetic peripheral neuropathy.

elevated NSE levels in the blood. ${ }^{13}$ Therefore, serum NSE can sensitively reflect the damage of neurons and brain tissue. Injury of neurovascular units is one of the main pathological mechanisms of diabetic cognitive impairment. Imaging evidence showed that white matter hyperintensities (WMH) in patients with type 2 diabetes were associated with cognitive dysfunction. ${ }^{14} \mathrm{WMH}$ is recognized as a marker of cerebral microvascular disease. Cerebral microvascular damage caused by diabetes may increase the susceptibility of neurons to ischemia and hypoxia, which eventually leads to neuron apoptosis. ${ }^{15}$ As we all know, DR is a neurovascular disease. ${ }^{16}$ Importantly, high glucose-induced retinopathy is strongly related to brain microvasculature. ${ }^{3}$ Retinal injury may cause cerebral arteriovenous shunt, resulting in malnutrition of nerve tissue and reduction of cerebral blood flow. This may make neurons more vulnerable to injury caused by ischemia and hypoxia. ${ }^{16}$ It is urgent to find an effective marker to screen cognitive impairment in DR patients.

In this study, we found that the serum NSE levels of patients in the MCI group were significantly higher than those in the normal cognition group. Moreover, high levels of serum NSE were associated with the risk of MCI among patients with DR. It is worth noticing that a previous study confirmed that serum NSE levels were associated with DPN. ${ }^{17}$ Given that the increase in serum NSE may be caused by many reasons, ${ }^{18}$ which may affect the relationship between NSE and MCI, we ran sensitivity analysis to test the robustness of the relationship between serum NSE and MCI. However, there were no statistically significant interactions between NSE and the relevant subgroups. This may indicate that the diabetes duration, the increase in HbAlc, the degree of DR, and the occurrence of DPN do not affect the relationship between serum NSE and MCI. These findings were similar to previous studies. Baranyi and Jones found that high levels of serum NSE were closely related to cognitive impairment in the elderly after surgery. ${ }^{7,8}$ Schmidt et al found significantly increased NSE in the CSF of Alzheimer's disease (AD) patients, and NSE was positively correlated with tau. ${ }^{19}$ Tau hyperphosphorylation, an important pathological feature of $\mathrm{AD}$, which may lead to cognitive dysfunction. ${ }^{20}$ Tau hyperphosphorylation has also been confirmed to be involved in the occurrence of diabetic cognitive dysfunction. ${ }^{21}$ Based on these findings, NSE may be associated with cognitive impairment, but the underlying mechanism of this relationship is unclear. Further researches based on human and animal models are helpful to elucidate the molecular mechanism between the two. In addition, the present study has shown that serum NSE can highly sensitively identify MCI in patients with DR. Therefore, serum NSE may be a powerful biomarker for predicting $\mathrm{MCI}$ in patients with DR.

Although diabetes has been proved to be related to cognitive impairment, the relationship between HbA1c and cognitive impairment is still controversial. An epidemiological study from Denmark showed that high HbAlc levels increased the risk of cognitive impairment. ${ }^{22}$ Furthermore, another study from Japan also confirmed that there was a potential trend of association between high $\mathrm{HbAlc}$ and cognitive impairment in elderly people with $\mathrm{T} 2 \mathrm{DM} .^{23}$ In line with this, this study found that the 


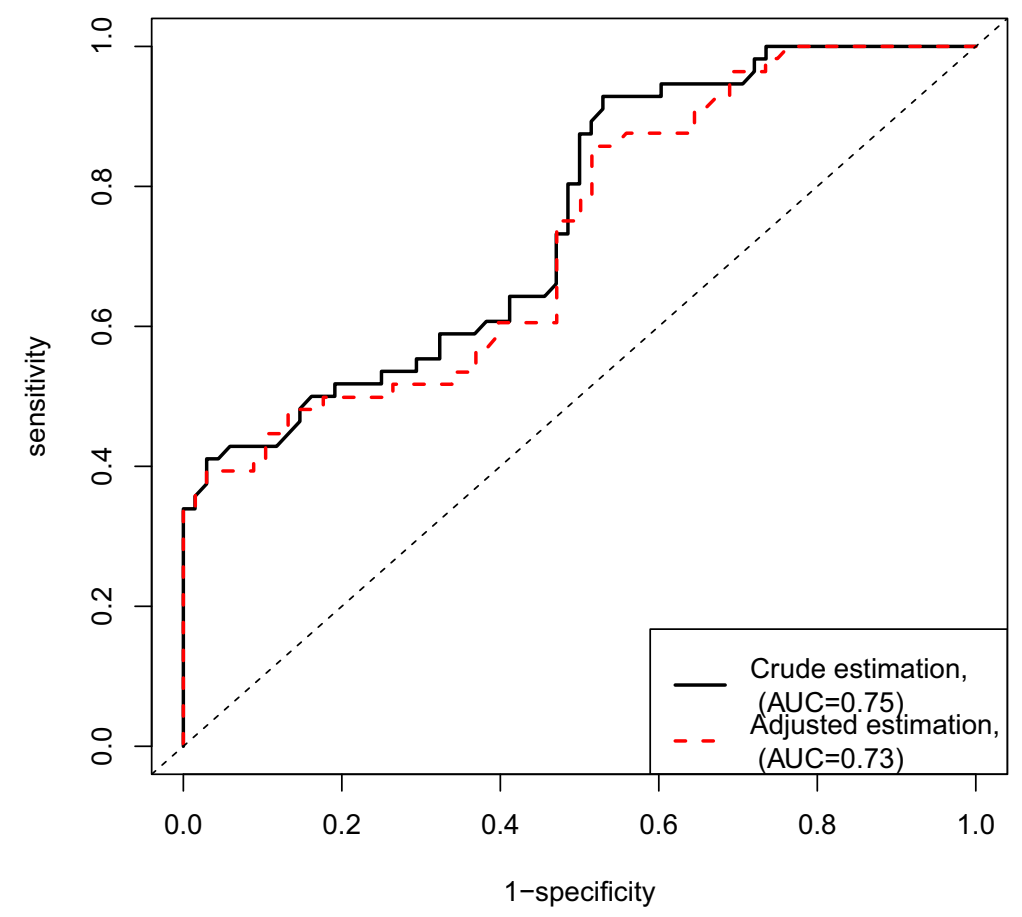

Figure I ROC curves for NSE.

Note: Adjusted estimation: adjusted for age, sex, education level, and HbAlc.

participants in the MCI group had higher HbAlc levels than those in the control group. These reports suggest that strict control of blood glucose levels is helpful to delay cognitive impairment. However, researchers found that there was no correlation between high $\mathrm{HbA} 1 \mathrm{c}$ levels and cognitive impairment in elderly T2DM patients from southern China. ${ }^{24}$ A study based on Korean elderly patients with T2DM showed that the average blood glucose levels were not related to cognitive decline. ${ }^{25}$ This contradiction may be due to the different countries, races and wealth levels of the subjects. In addition, there are currently no studies to discuss the relationship between HbA1c and cognitive impairment in the DR population. Longitudinal studies with larger sample sizes will be needed to clarify this potential link.

There are some deficiencies in this study. First, due to the cross-sectional nature of the study, the causal relationship between serum NSE and MCI cannot be inferred. Second, insufficient sample size and a single race may limit the application of this finding in other populations. Third, although MMSE scale is a widely used screening tool for cognitive impairment, it does not have enough sensitivity and specificity to identify MCI. ${ }^{26}$ In addition, MMSE score is easily affected by age and education level. Finally, no follow-up is designed in this study, and an additional follow-up period may be beneficial to further improve the relevant studies.

\section{Conclusion}

In conclusion, this study demonstrates that high levels of serum NSE are independently associated with MCI in patients with DR. Serum NSE is a potential predictor of MCI among DR patients.

\section{Acknowledgments}

This work was supported by a grant from the Natural Foundation of Heilongjiang Province [grant numbers H2015057, China].

\section{Disclosure}

Rong Liu is currently affiliated with the Department of Endocrinology, JiaoZuo People's Hospital, JiaoZuo, 454150, China. The authors report no conflicts of interest in this work.

\section{References}

1. Patterson $\mathrm{C}$ The state of the art of dementia research: new frontiers. An analysis of prevalence, incidence, cost and trends. Alzheimer's Disease International. World Alzheimer Report 2018. 2018.

2. Biessels GJ, Strachan MWJ, Visseren FLJ, Kappelle LJ, Whitmer RA. Dementia and cognitive decline in type 2 diabetes and prediabetic stages: towards targeted interventions. Lancet Diabet Endocrinol. 2014;2(3):246-255. doi:10.1016/S2213-8587(13)70088-3

3. Gupta P, Gan ATL, Man REK, et al. Association between diabetic retinopathy and incident cognitive impairment. $\mathrm{Br} J$ Ophthalmol. 2019;103(11):1605-1609. doi:10.1136/bjophthalmol-2018-312807 
4. Hafner A, Glavan G, Obermajer N, Živin M, Schliebs R, Kos J. Neuroprotective role of $\gamma$-enolase in microglia in a mouse model of Alzheimer's disease is regulated by cathepsin X. Aging Cell. 2013;12 (4):604-614. doi:10.1111/acel.12093

5. Li J, Yan M, Zhang Y, Xie M, Yan L, Chen J. Serum neuron-specific enolase is elevated as a novel indicator of diabetic retinopathy including macular oedema. Diabet Med. 2015;32(1):102-107. doi:10.1111/dme.12597

6. Asadova V, Gul Z, Buyukuysal RL, Yalcinbayir O. Assessment of neuron-specific enolase, S100B and malondialdehyde levels in serum and vitreous of patients with proliferative diabetic retinopathy. Int Ophthalmol. 2020;40(1):227-234. doi:10.1007/ s10792-019-01175-9

7. Jones EL, Gauge N, Nilsen OB, et al. Analysis of neuron-specific enolase and $\mathrm{S} 100 \mathrm{~B}$ as biomarkers of cognitive decline following surgery in older people. Dement Geriatr Cogn Disord. 2012;34(5-6):307-311. doi:10.1159/000345538

8. Baranyi A, Rothenhausler HB. The impact of S100b and persistent high levels of neuron-specific enolase on cognitive performance in elderly patients after cardiopulmonary bypass. Brain Inj. 2013;27 (4):417-424. doi:10.3109/02699052.2012.750751

9. Silva FP, Schmidt AP, Valentin LS, et al. S100B protein and neuron-specific enolase as predictors of cognitive dysfunction after coronary artery bypass graft surgery: a prospective observational study. Eur J Anaesthesiol. 2016;33(9):681-689. doi:10.1097/EJA.00 00000000000450

10. Alberti KG, Zimmet PZ. Definition, diagnosis and classification of diabetes mellitus and its complications. Part 1: diagnosis and classification of diabetes mellitus provisional report of a WHO consultation. Diabet Med. 1998;15(7):539-553. doi:10.1002/(SICI) 1096-9136(199807)15:7<539::AID-DIA668>3.0.CO;2-S

11. Albert MS, DeKosky ST, Dickson D, et al. The diagnosis of mild cognitive impairment due to Alzheimer's disease: recommendations from the National Institute on Aging-Alzheimer's Association workgroups on diagnostic guidelines for Alzheimer's disease. Alzheimers Dement. 2011;7(3):270-279. doi:10.1016/j.jalz.2011.03.008

12. Tsai JC, Chen CW, Chu H, et al. Comparing the sensitivity, specificity, and predictive values of the montreal cognitive assessment and mini-mental state examination when screening people for mild cognitive impairment and dementia in Chinese population. Arch Psychiatr Nurs. 2016;30(4):486-491. doi:10.1016/j.apnu.2016. 01.015

13. Sieber M, Dreßler J, Franke H, Pohlers D, Ondruschka B. Postmortem biochemistry of NSE and S100B: a supplemental tool for detecting a lethal traumatic brain injury? J Forensic Leg Med. 2018;55:65-73. doi:10.1016/j.jflm.2018.02.016
14. Mankovsky B, Zherdova N, van den Berg E, Biessels GJ, de Bresser J. Cognitive functioning and structural brain abnormalities in people with Type 2 diabetes mellitus. Diabet Med. 2018;35 (12):1663-1670. doi:10.1111/dme.13800

15. Umegaki $H$. Type 2 diabetes as a risk factor for cognitive impairment: current insights. Clin Interv Aging. 2014;9:1011-1019. doi:10.2147/CIA.S48926

16. Lu X, Gong W, Wen Z, Hu L, Peng Z, Zha Y. Correlation between diabetic cognitive impairment and diabetic retinopathy in patients with T2DM by (1)H-MRS. Front Neurol. 2019;10:1068. doi:10.3389/fneur.2019.01068

17. Li J, Zhang H, Xie M, Yan L, Chen J, Wang H. NSE, a potential biomarker, is closely connected to diabetic peripheral neuropathy. Diabetes Care. 2013;36(11):3405-3410. doi:10.2337/dc13-0590

18. Isgrò MA, Bottoni $\mathrm{P}$, Scatena $\mathrm{R}$. Neuron-specific enolase as a biomarker: biochemical and clinical aspects. Adv Exp Med Biol. 2015;867:125-143.

19. Schmidt FM, Mergl R, Stach B, Jahn I, Gertz HJ, Schonknecht P. Elevated levels of cerebrospinal fluid neuron-specific enolase (NSE) in Alzheimer's disease. Neurosci Lett. 2014;570:81-85. doi:10.1016/ j.neulet.2014.04.007

20. Yuan XY, Wang XG. Mild cognitive impairment in type 2 diabetes mellitus and related risk factors: a review. Rev Neurosci. 2017;28 (7):715-723. doi:10.1515/revneuro-2017-0016

21. Luchsinger JA. Type 2 diabetes and cognitive impairment: linking mechanisms. J Alzheimers Dis. 2012;30(Suppl 2):S185-198. doi:10.3233/JAD-2012-111433

22. Wium-Andersen IK, Rungby J, Jorgensen MB, Sandbaek A, Osler M, Wium-Andersen MK. Risk of dementia and cognitive dysfunction in individuals with diabetes or elevated blood glucose. Epidemiol Psychiatr Sci. 2019;29:e43. doi:10.1017/S2045796019000374

23. Umegaki H, Iimuro S, Shinozaki T, et al. Risk factors associated with cognitive decline in the elderly with type 2 diabetes: pooled logistic analysis of a 6-year observation in the Japanese elderly diabetes intervention trial. Geriatr Gerontol Int. 2012;12(Suppl 1):110-116. doi:10.1111/j.1447-0594.2011.00818.x

24. Huang L, Yang L, Shen X, Yan S. Relationship between glycated hemoglobin A1c and cognitive function in nondemented elderly patients with type 2 diabetes. Metab Brain Dis. 2016;31(2):347-353. doi:10.10 07/s11011-015-9756-Z

25. Kim C, Sohn J-H, Jang MU, et al. Association between visit-to-visit glucose variability and cognitive function in aged type 2 diabetic patients: a cross-sectional study. PLoS One. 2018;35(12):e0132118. doi:10.1371/journal.pone.0132118

26. Langa KM, Levine DA. The diagnosis and management of mild cognitive impairment: a clinical review. JAMA. 2014;312 (23):2551-2561. doi:10.1001/jama.2014.13806

Diabetes, Metabolic Syndrome and Obesity: Targets and Therapy

Dovepress

\section{Publish your work in this journal}

Diabetes, Metabolic Syndrome and Obesity: Targets and Therapy is an international, peer-reviewed open-access journal committed to the rapid publication of the latest laboratory and clinical findings in the fields of diabetes, metabolic syndrome and obesity research. Original research, review, case reports, hypothesis formation, expert opinion and commentaries are all considered for publication. The manuscript management system is completely online and includes a very quick and fair peer-review system, which is all easy to use. Visit http://www.dovepress.com/testimonials.php to read real quotes from published authors. 